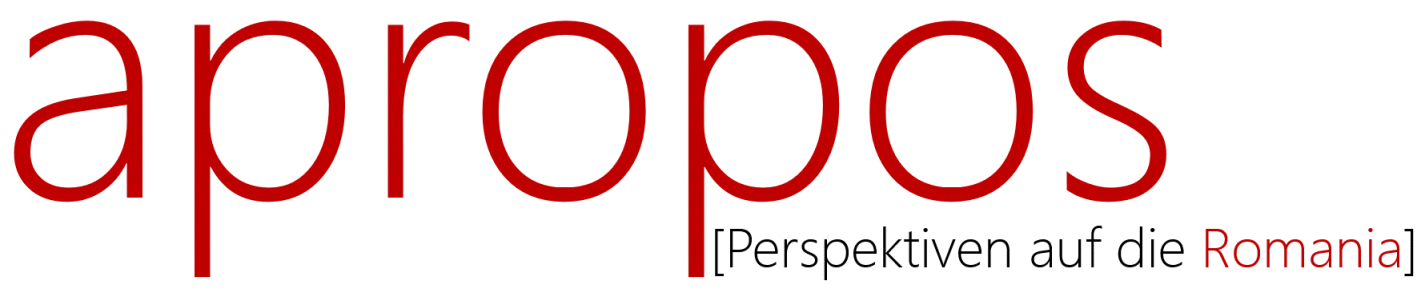

Sprache/Literatur/Kultur/Geschichte/Ideen/Politik/Gesellschaft

3 | 2019

Beziehungsweise(n)

Rezension von Mallet, Audrey. 2019. Vichy contre Vichy. Une capitale sans mémoire. Paris : Belin.

Pascal Gibert

apropos [Perspektiven auf die Romania]

hosted by Hamburg University Press

2019, 3

STAATS- UND UNIVERSITĀTS

BIBLIOTHEK

H A M B UR G

CARL VON OSSIETZKY

pp. 143-146.

ISSN: $2627-3446$

$\mathrm{DOI}$

https://doi.org/10.15460/apropos.3.1471

Zitierweise

Gibert, Pascal. 2019. „Rezension von Mallet, Audrey. 2019. Vichy contre Vichy. Une capitale sans mémoire. Paris: Belin“, apropos [Perspektiven auf die Romania] 3, 143-146. doi: 10.15460/apropos.3.1471

Except where otherwise noted, this article is licensed under a Creative Commons Attribution 4.0 International license (CC BY 4.0)

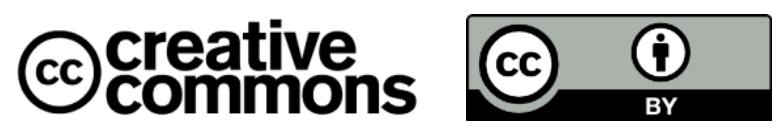


$2019, n^{\circ} 3$

pp. $143-146$

doi: 10.15460/apropos.3.1471

\title{
Pascal Gibert
}

\section{Compte rendu}

\section{MALLET, Audrey. 2019. Vichy contre Vichy. Une capitale sans mémoire. Paris : Belin.}

\author{
Pascal Gibert \\ est professeur d'histoire en Classes \\ Préparatoires aux Grandes Écoles \\ (hypokhâgne et khâgne) au Lycée \\ Mme de Staël à Montluçon (Allier). \\ gaugib@club-internet.fr
}

Mots-clés

Vichy - mémoire - État français - collaboration - années noires - épuration - Guerre d’Algérie

On connaît le combat - toujours actuel - de nombreux élus de la ville de Vichy consistant à vouloir substituer dans le langage commun l'expression de " régime de l'État français » à celle de "régime de Vichy ». L'ombre portée de la Seconde Guerre mondiale et de l'implantation de la capitale politique de la Collaboration pèse lourdement sur l'ancienne ville thermale, faisant d'elle un "non-lieu de mémoire ». L'ouvrage d'Audrey Mallet, issu de sa thèse de doctorat soutenue en 2016, propose une synthèse très utile abordant les différentes facettes des rapports que Vichy entretient avec son histoire et sa mémoire. Vichyssoise d'origine, l'auteure s'interroge sur les raisons du mutisme mémoriel local alors que la France est entrée dans l'ère de la mémoire; en quoi Vichy peut-elle se différencier à ce point des autres villes françaises ? La thèse d'Audrey Mallet a été codirigée par Norman Ingram, historien spécialiste de l'histoire du pacifisme dans l'entre-deux-guerres, et Henry Rousso, dont les travaux pionniers consacrés au "syndrome de Vichy » ont fait entrer la dimension mémorielle dans le champ de l'analyse historique. S'intégrant dans une démarche micro-historique, l'histoire et la mémoire de Vichy sont étudiées de manière à pouvoir saisir des processus plus amples. II ne s'agit donc pas de dresser une chronique historique de la ville mais de voir à travers elle comment s'articulent histoire et mémoire à plusieurs échelles. Contrairement à des représentations parfois ancrées dans les esprits, les sources disponibles sont abondantes: Archives nationales, Centre de Documentation Juive Contemporaine, Archives départementales et municipales, sources orales, etc.

Le premier chapitre de facture classique jette les bases d'un portrait bien connu de Vichy, " reine des villes d'eaux ». On sait ce que la ville doit au XIXe siècle et en particulier au Second Empire, succès qui survit au régime et qui s'intensifie au 
début du XXe siècle. Audrey Mallet envisage ensuite Vichy comme " capitale » et décrit une ville, dont la population locale a quintuplé en quelques semaines en 1940, qui devient le centre décisionnel de l'État français. Bénéficiant d'un sort économique un peu plus enviable que le reste du pays (ravitaillement plus fréquent, emploi de 2500 personnes dans l'industrie hôtelière locale, etc.), la ville est le lieu de la mise en scène permanente du régime. S'appuyant sur les apports décisifs de l'historiographie consacrée à l'étude de l'opinion publique, Audrey Mallet analyse les évolutions du positionnement des Vichyssois : si à l'instar du pays, ils ont été globalement passifs, leur passivité semble " particulièrement élevée ", les habitants de la ville apparaissant comme spectateurs de l'Histoire en marche. Par ailleurs, on retrouve dans la municipalité de Vichy, notamment chez son maire Pierre-Victor Léger, l'ambivalence décrite par Pierre Laborie dans ses travaux.

Le quatrième chapitre envisage une question peu étudiée jusqu'alors, celle des populations juives à Vichy. Les manifestations d'antisémitisme y semblent peu nombreuses dans l'entre-deux-guerres et lors de l'exode de 1940, Vichy reçoit de nombreux réfugiés juifs ainsi que des structures telles que le consistoire juif du BasRhin. Vichy se singularise à l'automne 1940 par une vague d'expulsions massives avant même les principales lois antisémites; le maréchal Pétain décide d' « épurer la ville de Vichy » et $95 \%$ des 3500 étrangers expulsés entre août et octobre sont juifs. Cette évolution particulière à la ville se poursuit : la diminution du nombre de la population juive est beaucoup plus importante que dans des villes comparables ; en 1941, sur les 2050 juifs qui se font recenser à Vichy, 88 \% sont français. Leur situation ne cesse d'empirer : 144 juifs résidant à Vichy sont déportés, 123 d'entre eux étaient français et 13 des Vichyssois d'avant-guerre.

À la Libération, on pouvait s'attendre à une épuration importante dans la capitale de l'État français. La mise en place rapide d'une cour martiale par les pouvoirs publics issus de la Résistance évite une épuration extrajudiciaire incontrôlée même si elle ne peut empêcher une phase de lynchages en juin 1945 au retour des prisonniers et des déportés. La présence de l'appareil de l'État français à Vichy entraîne à l'inverse une surreprésentation des habitants de cette ville dans le cadre de l'épuration judiciaire, notamment de la Cour de justice de l'Allier où ils représentent $52 \%$ des cas examinés. II semble bien que cela concerne particulièrement la «population temporaire » de Vichy - c'est-à-dire celle qui s'y est installée à partir de l'été 1940.

Dès lors il s'agit pour Vichy d' " effacer définitivement la souillure » des Années noires. C'est ce à quoi s'emploient les Vichyssois dès les premiers mois suivant la Libération. En commémorant les héros locaux, ils tentent de faire partie du grand récit mémoriel national. Peine perdue, le général De Gaulle ne vient pas à Vichy alors qu'il assiste à d'autres cérémonies comme à Moulins, préfecture de l'Allier. Vichy développe alors un discours mémoriel triplement victimaire : victime du choix de 1940 pour être le siège d'une capitale de substitution, victime des Allemands et victimes du régime de l'État français. Le sentiment d'un ostracisme de la part des 
autorités nationales domine chez les Vichyssois. La ville souhaite tourner la page alors que l'on assiste à une " reprise fulgurante du tourisme thermal » dès le début des années 1950. Une chape de plomb vient couvrir le souvenir de la guerre.

Les chapitres six et sept consacrés au rapport entre Vichy et la guerre d'Algérie apportent un éclairage très précieux sur une période peu connue mais essentielle pour la compréhension de l'évolution de l'histoire et de la mémoire locales. Audrey Mallet dresse le portrait d'une ville une nouvelle fois en décalage avec l'évolution du pays. Du fait de l'importance des relations étroites avec l'Empire colonial, la décolonisation semble sonner le glas de la cité. Cette dernière accueille les partisans de l'Algérie française, eux-mêmes porteurs d'une forte mémoire pétainiste. Mémoires et enjeux politiques immédiats s'entrechoquent. Autour des réunions de soutien à l'Algérie française qui connaissent une forte fréquentation, gravitent entre autres Jacques Isorni, l'avocat de Philippe Pétain, Raoul Salan et Jean-Marie Le Pen. Après la mort du Maréchal, la mémoire pétainiste, politique pour les uns, plus affective pour les autres, se développe de manière discrète dans les années 1950 puis de manière plus affirmée à partir des années 1960. Les thuriféraires du régime de l'État français, regroupés dans les rangs de l'Association pour Défendre la mémoire du Maréchal Pétain (ADMP) peuvent à l'occasion faire visiter l'appartement du Maréchal à des personnes triées sur le volet. II faut même une mobilisation des résistants locaux pour s'opposer à la création d'un musée sur Pétain à l'Hôtel du Parc en 1960. Face à cela, la réaction de la municipalité se limite en une tentative de réarmer le discours victimaire.

Alors qu'au niveau national les années 1980 sont celles du « retour du refoulé » des années noires qui confine à " l'obsession » (Henry Rousso), c'est le " silence local » à Vichy qui parait allier repli et refus. À l'heure des injonctions du « devoir de mémoire ", la mémoire locale est toujours en décalage, faisant de la ville de Vichy une exception au plan national, une " anomalie » selon l'auteure. On pourrait compléter cette étude de l'absence de mémoire visible par le travail d'HenriFerréol Billy sur un marqueur mémoriel quantifiable, à savoir le nombre de voies publiques baptisées de noms de résistants. Le verdict semble là aussi sans appel : vingt noms de rues et de places à Montluçon, autre grande ville de l'Allier à la forte mémoire résistante, onze pour la préfecture, Moulins, et seulement quatre pour Vichy!

De nouveaux instruments et de nouvelles structures, permettant une histoire et une mémoire plus distanciées en marge de la politique mémorielle institutionnelle, sont fort heureusement aujourd'hui disponibles: depuis 2016, le Centre International d'Études et de Recherches de Vichy (CIERV) organise localement des conférences d'historiens de premier plan, diffusant les résultats des recherches récentes. Par ailleurs, Audrey Mallet est l'auteure de l'application mobile « Vichy 1939-1945 ", disponible gratuitement sur les plates-formes de diffusion, qui rend possible une visite virtuelle bien documentée de la ville des Années noires. II s'agit là d'œuvres utiles. 


\section{Bibliographie}

BILLY, Henri-Ferréol. 2015. "Résistants et noms de rue dans I'Allier », $<$ http://histoire-et-genealogie.over-blog.com/2015/08/resistants-etnoms-de-rue-allier.html>, 20.12.2019.

GIBERT, Pascal. 2019. " Une épuration particulière ? La répression de la collaboration à Vichy et dans sa région (1944-1946) ", Siècles [En ligne], 47/2019, <http://journals.openedition.org/siecles/5664>, 20.12.2019

LABORIE, Pierre. 1990 [2001²]. L'opinion française sous Vichy. Les Français et la crise d'identité nationale, 1936-1944. Paris : Points Histoire.

LABORIE, Pierre. 2014. Le chagrin et le venin. Occupations, résistance. Idées reçues. Paris : Folio.

LABORIE, Pierre. 2019. Penser l'événement. 1940-1945. Édition de Jean-Marie Guillon et Cécile Vast, Paris : Gallimard.

Rousso, Henry. 1990. Syndrome de Vichy : De 1944 à nos jours. Paris : Points Seuil. 